\title{
The effect of mesenchymal stem cells' secretome on lung cancer progression is contingent on their origin: primary or metastatic niche
}

\author{
Oshrat Attar-Schneider ${ }^{1,2,3} \cdot$ Liat Drucker ${ }^{2,4} \cdot$ Maya Gottfried ${ }^{1,3,4}$
}

Received: 17 April 2018 / Revised: 2 July 2018 / Accepted: 4 July 2018 / Published online: 8 August 2018

(c) United States \& Canadian Academy of Pathology 2018

\begin{abstract}
The fatality of non-small-cell lung cancer (NSCLC) and the role of the cancer microenvironment in its resistance to therapy are long recognized. Accumulating data allocate a significant role for mesenchymal stem cells (MSCs) in the malignant environment. Previously, we have demonstrated that MSCs from NSCLC metastatic bone marrow (BM) niche deleteriously affected NSCLC cells. Here, we have decided to examine the effect of MSCs from the primary niche of the lung (healthy or adjacent to tumor) on NSCLC phenotype. We cultured NSCLC cell lines with healthy/NSCLC lung-MSCs conditioned media (secretome) and showed elevation in cells' MAPKs and translation initiation signals, proliferation, viability, death, and migration. We also established enhanced autophagy and epithelial to mesenchymal transition processes. Moreover, we observed that MSCs from tumor adjacent sites (pathological niche) exhibited a more profound effect than MSCs from healthy lung tissue. Our findings underscore the capacity of the lung-MSCs to modulate NSCLC phenotype. Interestingly, both tumor adjacent (pathological) and distant lung-MSCs (healthy) promoted the NSCLC's TI, proliferation, migration, and epithelial to mesenchymal transition, yet the pathological MSCs displayed a greater affect. In conclusion, by comparing the effects of normal lung-MSCs, NSCLC adjacent MSCs, and BM-MSCs, we have established that the primary and metastatic niches display opposite and critical effects that promote the cancerous systemic state. Specifically, the primary site MSCs promote the expansion of the malignant clone and its dispersion, whereas the metastatic site MSCs facilitates the cells reseeding. We suggest that sabotaging the cross-talk between MSCs and NSCLC affords effective means to inhibit lung cancer progression and will require different targeting strategies in accordance with niche/disease stage.
\end{abstract}

These authors contributed equally: Liat Drucker, Maya Gottfried

Electronic supplementary material The online version of this article (https://doi.org/10.1038/s41374-018-0110-z) contains supplementary material, which is available to authorized users.

Oshrat Attar-Schneider

oshrat.attar@clalit.org.il

1 Lung Cancer Research, Lung Cancer Unit, Meir Medical Center, Kfar Saba 44281, Israel

2 Oncogenetic Laboratories, Lung Cancer Unit, Meir Medical Center, Kfar Saba 44281, Israel

3 Department of Oncology, Lung Cancer Unit, Meir Medical Center, Kfar Saba 44281, Israel

4 Sackler Faculty of Medicine, Tel Aviv University Ramat Aviv, Tel Aviv 69978, Israel

\section{Introduction}

The genetics of lung cancer remain a therapeutic challenge [1]. In-depth analyses of lung cancer genomes have defined non-small-cell lung cancer (NSCLC) as a group of distinct diseases with genetic and cellular heterogeneity [2]. The updated concept of tumor heterogeneity applies not only to tumor epithelial cells but also to the diverse microenvironments with which the tumor cells interact. Multiple studies have established that cancer cells are closely associated with the extracellular matrix (ECM), various niche cell types, infiltrating immune cells, and vasculature [3]. In some cases, this environment is essential for tumor initiation or tumor growth, whereas in other cases, it can prevent tumorigenesis or even promote tumor clearance (reviewed in ref. [4]).

Interestingly, distinct types of cells such as mesenchymal stem cells (MSCs) contribute to these phenomena through a continuous cross-talk with the malignant cells directly or 
indirectly (secretome, ECM) [5]. MSCs are particularly interesting because of their great plasticity and versatile involvement in the malignant process [6]. They are a subset of adult stem cells that possess abilities of self-renewal and multilineage differentiation (chondrocytes, osteocytes, and adipocytes)[7]. Intriguingly and pertaining to our study, they are reported to both promote and inhibit cancer in multiple publications [8]. The mechanisms for this variability are currently far from deciphered and both the cancer cells and their surroundings are implicated. The long held hypothesis was that cancer metastases arise from rare primary tumor cells that sequentially acquire traits that allow them to invade through ECM, survive in the circulation, extravasate at distant sites, attach and proliferate at the new location. The recognition of the significant interaction with the tumor microenvironment adds another layer of regulation, yet much is unknown regarding the context and rules of the bidirectional involvement of MSCs in neoplasia. We hypothesized that the NSCLC cells may participate in different dialogues when exposed to MSCs from different surroundings [9].

It is commonly known that the bone marrow (BM) is a fertile soil for NSCLC tumor cells to create metastasis due to factors released into and by the bone residents [10]. Indeed, in our previous studies, we have delineated some basic and critical interactions between NSCLC and the BM niche. Specifically, have showed that BM-MSCs are able to suppress NSCLC cells' growth and elevate their death [11]. We have also demonstrated an inhibitory effect of BM-MSCs on lung cancer cells' migration in a MAPK signaling and by translation initiation (TI) contingent manner [11].

These findings piqued our interest and we wondered whether lung resident MSCs have the same effect on the malignant cells. We speculated that MSCs derived from the primary site may differ in their effect from MSCs of the metastatic site, namely, BM. Furthermore, we hypothesized that there may be differences between MSCs in close proximity to the tumor cells and those that originate from normal lung tissue. For this purpose, we cultured NSCLC cells with lung-MSCs' secretome and compared it to the effects we have witnessed with the BM-MSCs.

Previous studies by others and us have demonstrated that MSCs derived from pathological settings exhibit genetic and functional abnormalities compared to their normal counterparts [12]. Specifically, it was reported that MSCs in lung cancer tissue have demonstrated accelerated growth, and reduced sensitivity to drugs [13]. Therefore, we also examined and compared the effects of MSCs from normal lung tissue on NSCLC cells with the effects of MSCs derived from the tumor tissue.

As we have reported, TI regulates NSCLC cell lines' proteome and phenotype $[11,14]$. It is also well established that protein translation is particularly responsive to cues originating from the cells' microenvironment. Thus, in the current study, we also studied the MSCs' secretome effects on the TI status in the NSCLC cells.

Our observations support our conjecture that MSCs play a critical and dynamic role in NSCLC cancer and differ at the primary vs. metastasis site and normal vs. pathological environments.

\section{Materials and methods}

\section{NSCLC cell lines}

Authenticated H1299, H460, H1975, and A549 were cultured in 10\% FCS RPMI1640 (Biological Industries, Israel).

\section{Lung-MSCs isolation and propagation}

Healthy and tumor lung tissues were obtained from therapynaive patients who underwent lung surgery for newly diagnosed NSCLC (all donors signed informed consent form approved by the Meir medical center's Helsinki committee and complying with Helsinki regulations). Healthy tissue samples were taken from the most removed section of the dissected NSCLC carrying lobe $(n=14$, 8 females, 6 males-aged $62.9 \pm 4.4$ ). Samples were minced into $1 \mathrm{~mm}^{3}$ pieces and digested. Non-adherent cells were removed with the media of culture leaving the adhered MSCs in the culture dish. MSCs were propagated as we have done previously [11]. Next, the cells were tested for MSCs markers: positivity for vimentin (DakoCytomatoion, Denmark) and negativity for keratin (Zymed, CA, USA) by immunocytochemistry (Fig. 1a). The cells' multi-potency was tested by assessing their capacity to differentiate into adipocytes and/or osteoblasts using StemPro adipogenesis and osteogenesis differentiation kit (Invitorgene, Carlsbad, CA, USA). Adipogenic and osteogenic differentiations were demonstrated using Sudan IV staining and Alizarin Red (Sigma) staining, respectively (Fig. 1b). Presence of human MSCs markers $\mathrm{CD} 90^{+} / \mathrm{CD} 105^{+} / \mathrm{CD} 44^{+} / \mathrm{CD} 45^{-} / \mathrm{CD} 19^{-} /$ CD14 ${ }^{-} / \mathrm{CD}^{-} 4^{-}$(MACS, Miltenyi Biotec, Germany) by flow cytometry (Navios, Beckman Coulter, USA) (Fig. 1c) were assayed.

\section{Lung-MSCs' secretome model}

Lung-MSCs conditioned medium (secretome) was collected every $72 \mathrm{~h}$ from $80 \%$ conflunty MSCs' culture flasks. Upon experiment, the medium was mixed with fresh media (7:1 ratio, respectively) and applied to NSCLC cell lines. The cells' response was normalized to respective NSCLC secretome as described by us before [11]. 


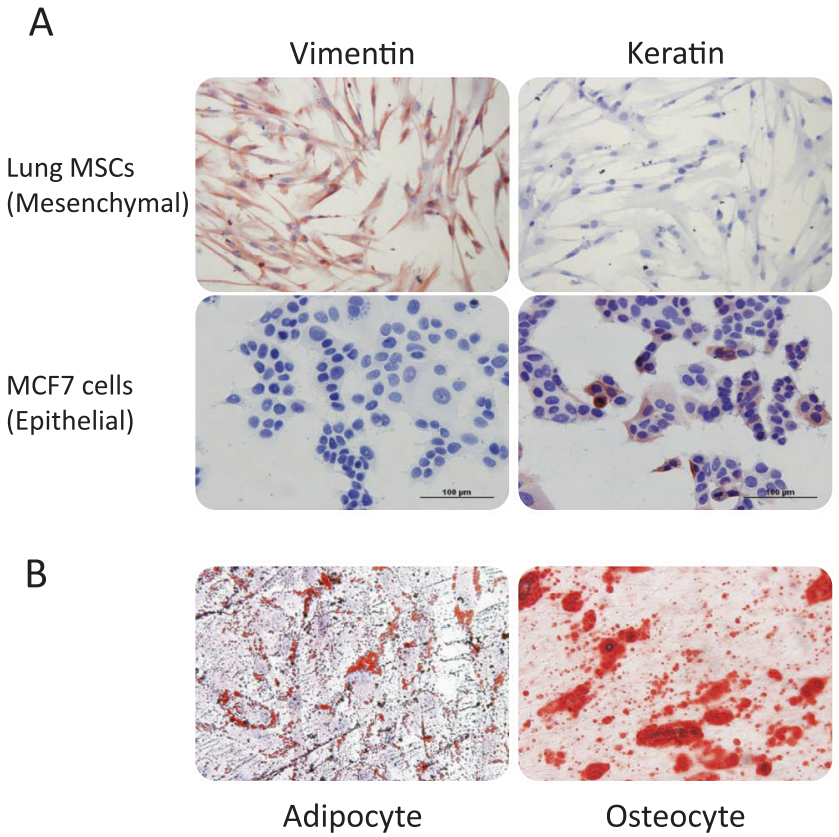

Fig. 1 Lung mesenchymal stem cells (MSCs) identification and characterization: lung-MSCs were seeded on round slides inserted into the culture wells $\left(1.9 \mathrm{~cm}^{2}\right)$ for $24 \mathrm{~h}$ and tested for a MSCs markers: positivity for vimentin and negativity for keratin by immunocytochemistry; b the cells' multi-potency was tested by assessing their

\section{BM-MSCs isolation and secretome model}

As described by us before [11]. Briefly, BM samples were obtained from femur head samples of normal donors undergoing elective full hip replacement due to orthopedic purposes (all donors signed informed consent form approved by the Meir medical center's Helsinki committee and complying with Helsinki regulations). Mononuclear cells were isolated from BM samples on Ficoll (Sigma, Israel). Non-adherent cells were removed from the media of culture, leaving the adhered MSCs in the culture dish. BMMSCs' conditioned medium (secretome) was collected every $72 \mathrm{~h}$ from MSC culture flasks with $80 \%$ confluence. Conditioned medium was centrifuged and upper fluids were collected into fresh tubes.

\section{Trypan blue}

Total cell counts as well as the respective proportion of live and dead cells were enumerated by Trypan blue dye exclusion using a Countess Automated cell counter and a phase-contrast microscope.

\section{Immunoblotting}

NSCLC cells were lysed and western blot was performed as described elsewhere [15]. The proteins were detected
C
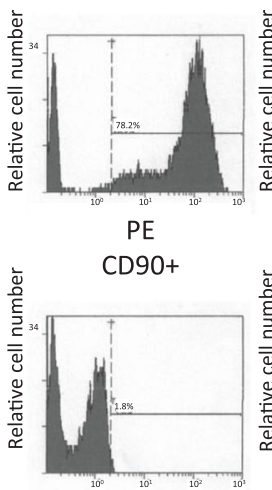

PE

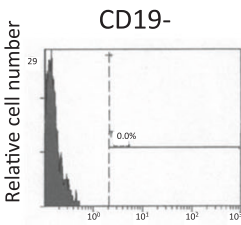

PE

CD45-

capacity to differentiate into adipocytes and/or osteoblasts. Adipogenic and osteogenic differentiations were demonstrated using Sudan IV staining and Alizarin Red staining, respectively. c Flow cytometry demonstrated presence/absence of human MSCs' markers: CD90 ${ }^{+} / \mathrm{CD} 105^{+} / \mathrm{CD} 44^{+} / \mathrm{CD} 45^{-} / \mathrm{CD} 19^{-} / \mathrm{CD} 14^{-} / \mathrm{CD} 34^{-}$

with rabbit/mouse anti-human: peIF4E(Ser209)/total eIF4E, peIF4GI(Ser1108)/total eIF4GI, p4EBP(Ser65)/total 4EBP, pmTOR(Ser2448)/total mTOR, pSAPK/JNK (Thr183/ Tyr185)/total JNK, phospho-p44/42 MAP kinase, ERK (Thr202/Tyr204)/total ERK, cyclin D1 (Cell Signaling, Danvers, MA, USA); pMNK(Thr197/Thr202)/total MNK, SMAD5, HSC70 (Epitomics, CA, USA); and HIF1 $\alpha$, NFkB (Santa-Cruz, CA, USA).

\section{Cell viability assay}

Assessment of viability was performed on NSCLC cell lines using cell proliferation reagent WST-1 (Roche) as described before [16].

\section{Scratch assay}

Were conducted as described before [17]. Wound closure was monitored by microscopy immediately after cell scratching $(0 \mathrm{~h})$ and at $24 \mathrm{~h}$ post wounding.

\section{Inhibitors}

MAPK inhibitors: SP600125 $(20 \mu \mathrm{M}$, JNK inhibitor, Biomol Int., USA), U0126 (10 $\mu \mathrm{M}$, MEK1/2 inhibitor, CST, USA), and 4EGI-1 (eIF4E/eIF4G Interaction Inhibitor, $35 \mu \mathrm{M}$, EMD Millipore). All were dissolved in DMSO. 


\section{MSCs secretome fractionation}

MSCs secretome was separated for two fractions with $100 \mathrm{kDa}$ cutoff by Amicon Ultra-15 centrifugal filter devices (Merck Millipore) according to manufactures' instructions. Briefly, MSCs secretome was applied into Amicon devices, and centrifuged (15-30 min, 4000×g) until 30 $\mu \mathrm{l}$ remain in the upper chambers. The separation procedure yielded a high-molecular weight fraction of $>100 \mathrm{kDa}$ and all media with remaining solubles as the lower molecular weight fraction $<100 \mathrm{kDa}$. Therefore, we used whole undivided MSCs' secretomes as control for the $<100 \mathrm{kDA}$ fraction. For the $>100 \mathrm{kDA}$ fraction that was suspended in low-serum $(3 \%)$ fresh media of identical volume as the original sample, we used low-serum fresh media as control.

\section{Statistical analysis}

Student's paired $t$ tests were used in analysis of differences between cohorts. Effects were considered significant when $p$-value is $<0.05$.

\section{Institutional Helsinki approval}

MSCs were isolated from donors' samples as approved by the Meir medical center's Helsinki committee and complying with Helsinki regulations.

\section{Results}

\section{Lung-MSCs' secretome induced NSCLC cells' viability and proliferation and inhibited cells' death}

Initially, we assessed the viability of NSCLC cell lines treated with normal or NSCLC lung-MSCs' secretomes with WST1 reagent and determined significantly elevated viability after $72 \mathrm{~h}$ (50-70\% $\uparrow$ normal lung; 80-120\% $\uparrow$ NSCLC, $p<0.05$ ) (Fig. 2a). Total and dead cell counts were also assessed. Our findings demonstrated significantly increased total cell counts $(65-100 \% \uparrow$ normal lung; 100-180\% $\uparrow$ NSCLC, $p<0.05 / 0.01$ ) (Fig. 2a), while examination of the death rates showed reduced percentages (20-60\% $\downarrow$ normal lung and NSCLC, $p<0.05$ ) (Fig. 2a). In order to determine whether the increased cell counts stemmed from changes in cell proliferation on top of reduced cell death, we assayed the expression of the proliferation marker PCNA. Indeed, elevated PCNA levels were determined in NSCLC cells exposed to lung-MSCs' secretomes (50-130\% $\uparrow$ normal lung; 90-215\% $\uparrow$ NSCLC, $p<0.01$ ) (Figs. 2b, 3a). Interestingly, although both healthy and NSCLC lung-MSCs stimulated NSCLC cells, the effects of the malignant secretome on viability, cell counts, and PCNA were significantly more profound $(p<0.05)$.

Of note, in our previous study, we have shown that BM-MSCs in contrast to lung-MSCs inhibited NSCLC cells' viability and proliferation and promoted cell death [11]. These results show for the first time that lung-MSCs (primary lung cancer niche) and BM-MSCs (representing the lung cancer metastatic niche) demonstrate opposite effects on NSCLC cell lines' phenotype.

\section{NSCLC cell lines treated with lung-MSCs' secretome showed augmented migration}

It is well established that microenvironmental factors are essential for cells' migration [18]. Thus, we assayed the migration capabilities of lung-MSCs' secretomes-treated NSCLC using scratch assay as well as assessment of MMPs' activity in zymogram. The scratch assay indicated that lung-MSCs' secretome caused a significant acceleration in the cells' migration (110-150\% $\uparrow$ normal lung; $170-250 \% \uparrow$ NSCLC of scratch closure, $p<0.01$ ) (Fig. 2c) compared to control cells. We also examined the cells' morphology following the secretome treatments. Indeed, the treated cells that demonstrated migrating phenotype exhibited a more fibroblast-like spindle appearance compared to untreated NSCLC cells that displayed an epithelial-like more round morphology (Fig. 2d). Here too, all our observations were significantly more prominent in the NSCLC-MSCs' secretome $(p<0.05)$. Again the effect of lung-MSCs secretomes on NSCLC cells migration was opposite to the influence of BM-MSCs secretome $(p<$ 0.05 ). Previous studies have shown that matrix metalloproteinases (MMPs) have an effect on the aggressive course of NSCLC [19, 20]. One of the major implications of MMPs in cancer progression is their role in paving the way through the tissue by ECM degradation, which allows cancer cells to migrate out of the primary tumor to form metastases [21]. Thus, we performed zymogram assay in order to quantify the levels of MMP9/MMP2 in the lungMSCs' secretome. Our measurements determined that there are significantly higher levels of MMP9/MMP2 levels in the lung-MSCs' secretome compared to cell-free medium (230-330\% $\uparrow, p<0.01)$ (Figs. 2e, 3b).

\section{Lung-MSCs' secretome induced $\mathrm{TI}$ and autophagy in NSCLC cell lines}

It is well acknowledged that protein synthesis is important to the cell's proliferation and migration [22-25]. Thus, once we established the changes in the cells' proliferation and migration, we further investigated protein synthesis-related pathways. Specifically, we examined the involvement of two major TI factors, eIF4E/eIF4GI and their regulators and 
A

Lung-MSCs' secretome induced NSCLC cells' viability and proliferation and inhibited cells' death

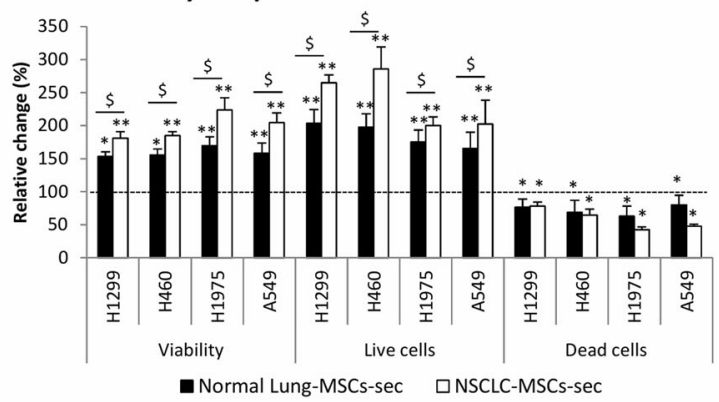

C
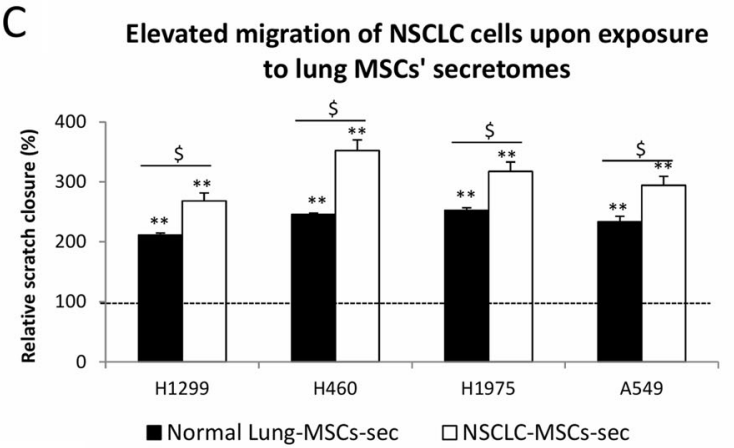

D
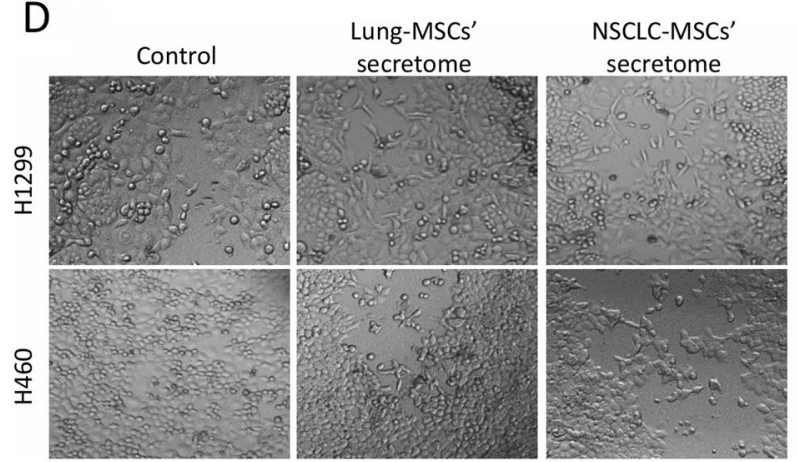

Fig. 2 NSCLC cells displays elevated viability, proliferation, migration, and MMPs' levels upon exposure to lung-MSCs' secretome: NSCLC cells were cultured with lung-MSCs secretome (normal/ NSCLC). After $72 \mathrm{~h}$, the cells were collected and assessed for changes in the cells' a viability/proliferation/death rate. Next, b treated NSCLC cell lines were lysed and immunoblotted for PCNA protein (proliferation marker). Immunoblot results are normalized to HSC-70 loading control $(n \geq 3)$ in bar graphs and representative immunoblots (Fig. 3a). c Lung-MSCs secretome effect on cell migration was assessed by scratch assay. Scratch closure were photographed

targets. In concordance with our expectations, analyses of the TI profile in NSCLC cell lines treated with lung-MSCs' secretome $(72 \mathrm{~h})$ indicated profoundly increased levels of phosphorylated and total eIF4E and eIF4GI (90-150\% $\uparrow$ normal lung; 105-240\% $\uparrow$ NSCLC, $p<0.05$ ) (Figs. 4a, 3c). We also determined a comprehensive activation of the treated cells' TI regulators (mTOR, MNK1/2, 4EBP) $(80-135 \% \uparrow$ normal lung; 95-195\% $\uparrow$ NSCLC, $p<0.05$ ) (Figs. 4a, 3c). In order to prove the functionality of the increased translational

\section{B Lung-MSCs' secretome induced NSCLC cells' PCNA signal}
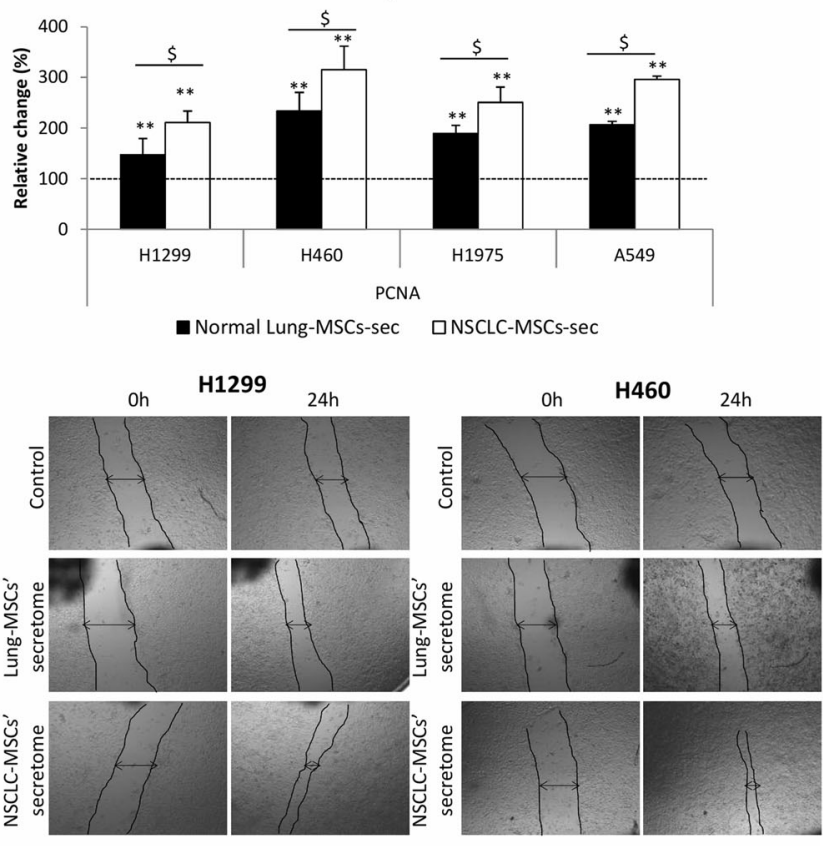

$E$

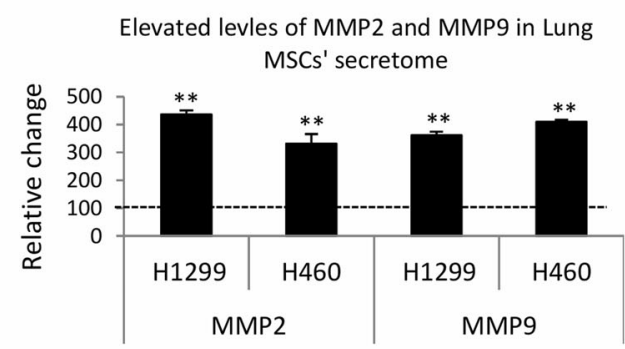

immediately $(0 \mathrm{~h})$ and after $24 \mathrm{~h}$ (magnification, $\times 40$ ). Results are presented as percent in bar graphs (left) and representative images of two lines (right). d Morphology changes of NSCLC cells treated with lung-MSCs' secretome (representative images of two lines). e MMP9/ MMP2 levels in lung-MSCs secretome were measured using zymogram assay. Results are presented as percent (mean $\pm \mathrm{SE}$ ) of control cells/media (dotted line) and representative images (Fig. 3b). Statistically significant differences between cohorts $(* p<0.05, * * p<0.01$; compare to control. ${ }^{\$}$ Normal vs. NSCLC) are indicated

activity, we also assessed the levels of important eIF4E/ eIF4GI's dependent proteins and observed significantly increased levels (NFkB, SMAD5, cyclin D, HIF1 $\alpha$ ) (75-135\% $\uparrow$ normal lung; $125-270 \% \uparrow$ NSCLC, $p<0.05$ ) (Figs. 4a, 3c). Again, all observations were significantly more prominent in the NSCLC cells treated with NSCLC-MSCs' secretome than normal lung-MSCs secretome $(p<0.05)$. Altogether, these observations indicate that the lung-MSCs' secretomes contained elements that increased translational 

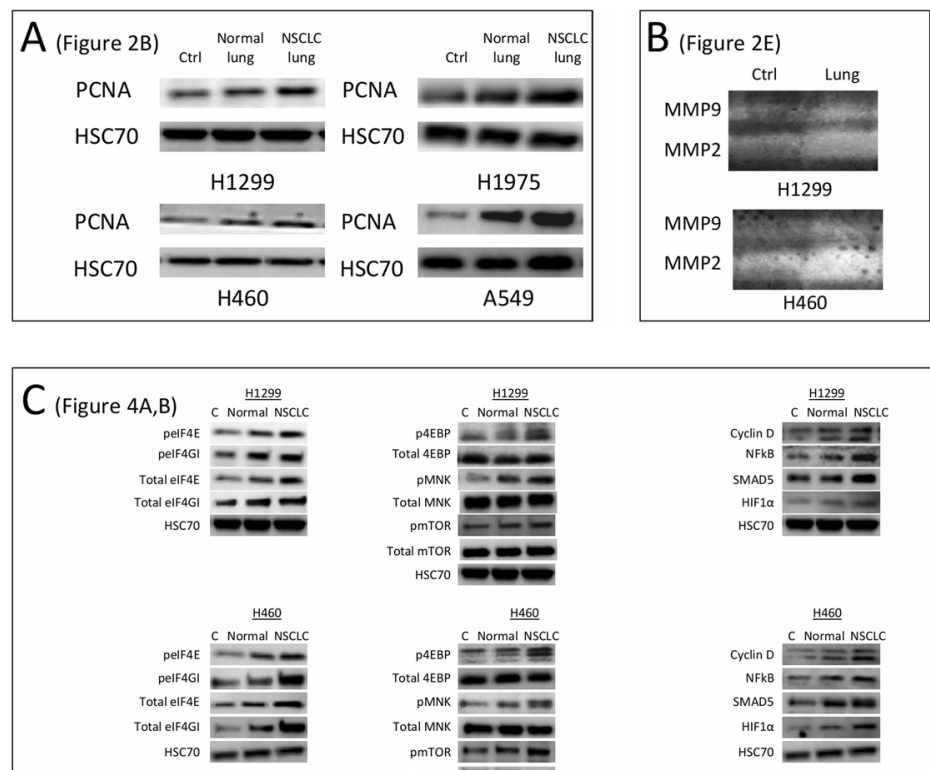
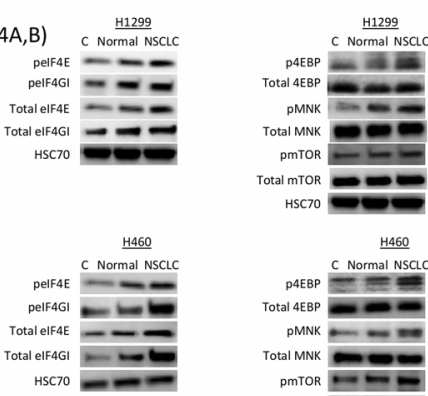

$=-$

tal MNK 5

HSC70

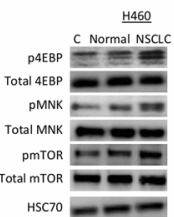

$\frac{H 460}{\text { NSCLC }}$

PMNK $-\cdots$

Tol MNK -2

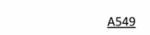

4549
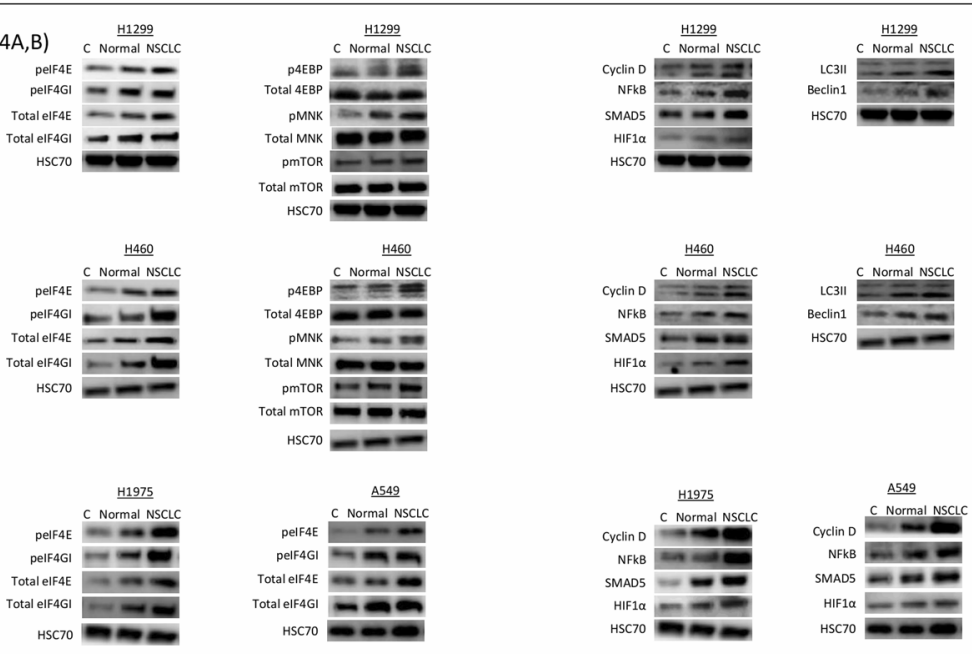

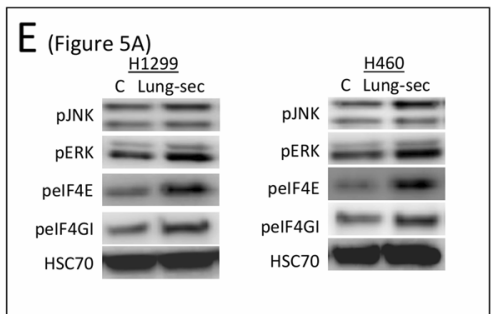

$F_{\text {(Figure 5B) }}$

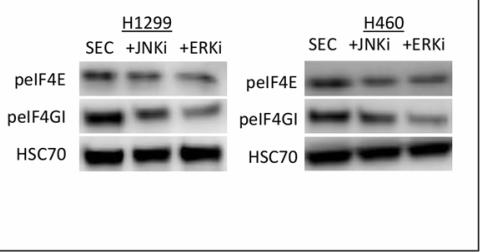

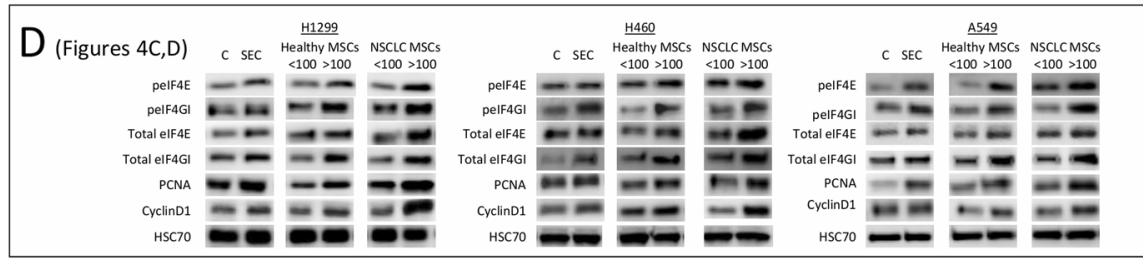

Fig. 3 Representative experiments' immunoblots: NSCLC cells were lysed and immonubloted. The figure demonstrates representative immunoblot images for each of the experiments as follow: a figure $2 \mathrm{~B}$ b figure $2 \mathrm{E}$ c figure $4 \mathrm{~B}, \mathrm{C} \mathbf{d}$ figure $4 \mathrm{C}, \mathrm{D}$ e figure $5 \mathrm{~A} \mathbf{f}$ figure $5 \mathrm{~B}$. activity of eIF4E and eIF4GI in NSCLC cells. These observations are again opposed to the ones we made and published regarding the effect of BM-MSCs secretomes modulation of TI [11] $(p<0.05)$.

The involvement of autophagy in NSCLC and its function in the cells' survival and proliferation is well established [26]. Therefore, we measured the activation of LC3II and Beclin, both essential for autophagosomes' formation, in NSCLC cell lines treated with lung-MSCs' secretomes. Interestingly, we observed increase in both autophagy markers (60-145\% $\uparrow$ normal lung; 130-275\% $\uparrow$ NSCLC, $p<$ 0.05 ) (Figs. 4b, 3c), while the effect we detected in NSCLC cells treated with MSCs' secretome originated from the tumor area was significantly higher $(p<0.05)$.

Finally, using centrifugal filtration with a semipermeable membrane of a $100 \mathrm{kDa}$ molecular weight cutoff (Centricon), we separated healthy lung-/NSCLC-MSCs' secretomes into a $<100 \mathrm{kDa}$ fraction that contained soluble proteins, cytokines, and exosomes $(<100 \mathrm{kDa})$ and a
$>100 \mathrm{kDa}$ fraction that contained large extracellular microvesicles (MVs) (>100 kDa). Surprisingly, while NSCLC cell lines treated with lung-MSCs secretomes' $<100 \mathrm{kDa}$ fraction did not show any significant changes, the cells treated with lung-MSCs secretomes $>100 \mathrm{kDa}$ fraction demonstrated significantly elevated levels in the cells' proliferation, migration, and TI status in concordance with the results of the un-separated conditioned media (Figs. 4c, $\mathrm{d}$ and $3 \mathrm{~d}$ ). These findings suggest that factors higher than $100 \mathrm{kDa}$ extracellular vesicles (EV/MV) are responsible for the effects caused by the lung-MSCs' secretomes.

\section{MAPK signaling regulates NSCLC TI and consequently migration in lung-MSCs secretome- treated NSCLC cells}

Previously, we have witnessed that BM-MSCs secretome induces changes in NSCLC phenotype/TI status (72 h) and earlier changes in cell migration $(6-24 \mathrm{~h})$ [11]. We have 

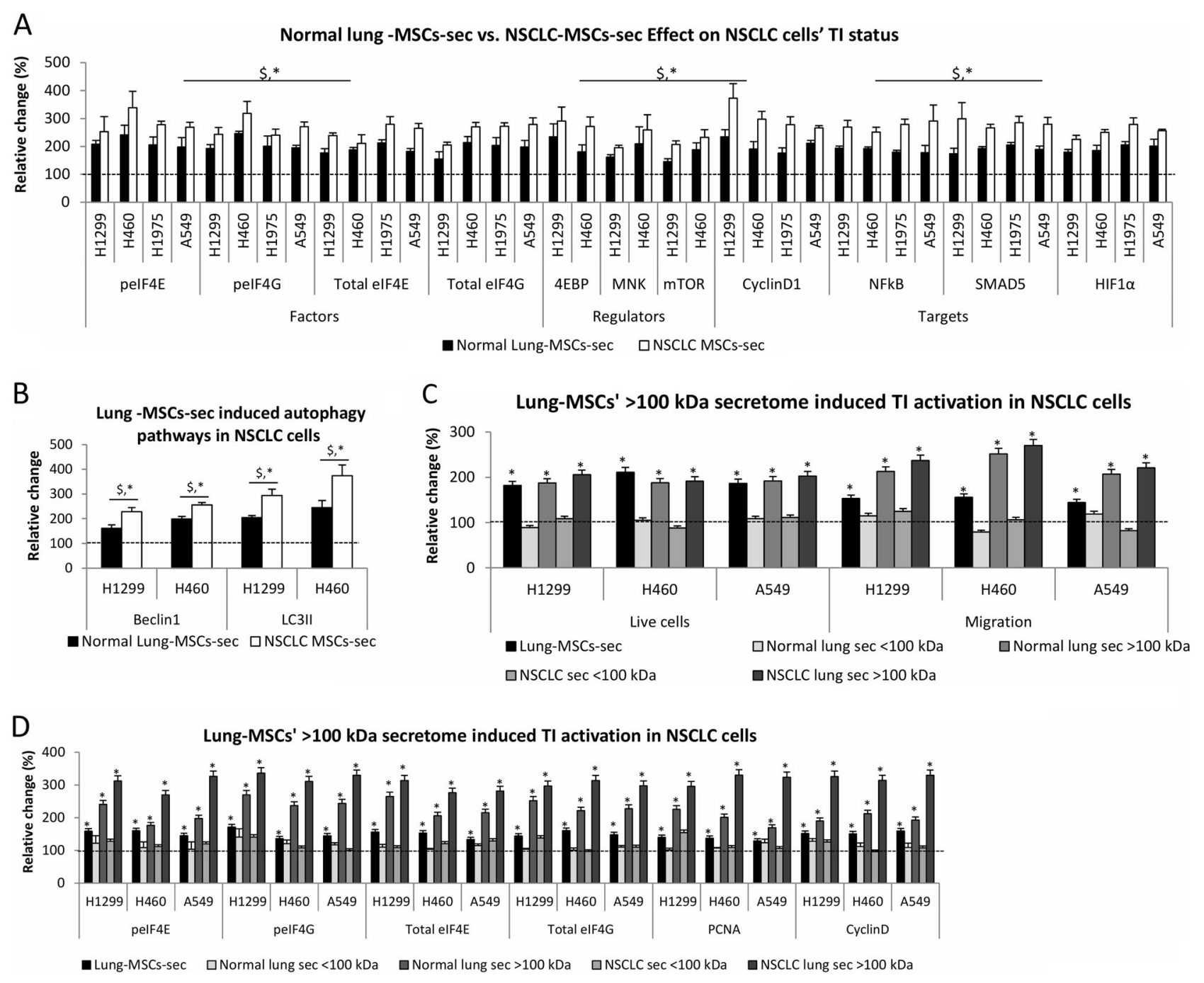

Fig. 4 BM-MSCs secretome upregulated both translation initiation and autophagy signals: NSCLC cells were cultured with lung-MSCs secretome (normal/NSCLC). Following $72 \mathrm{~h}$ of culturing, the cells were lysed and immonubloted for a TI factors eIF4E/eIF4GI, the factors' regulators: 4EBP, MNK, mTOR; and targets: eIF4E (NFkB, cyclin D1), eIF4GI (SMAD5), both (HIF1 $\alpha$ ). b Cells were also immonubloted for the autophagy markers LC3II and Beclin1. Next, NSCLC cells were cultured for $72 \mathrm{~h}$ with fresh low-serum media containing $>100 \mathrm{kDa}$ fraction or lacking the $>100 \mathrm{kDa}$ of lung-MSCs

discovered that MAPK (ERK, JNK) signals were responsible for the early effects since they are an earlier upstream regulator of both migration and TI [27] and regulate the upstream regulators of eIF4E and eIF4GI [28]. Here, we wanted to verify whether lung-MSCs' secretome induce similar cascades and time course of events.

Thus, we cultured NSCLC cell lines with lung-MSCs' secretome for $1.5 \mathrm{~h}$ and assayed the levels of active phosphorylated forms of MAPKs, ERK, and JNK. Indeed, elevated levels of pJNK and pERK were observed (pJNK: 120\% $\uparrow$; pERK: $110 \% \uparrow ; ~ p<0.01$ ) (Figs. 5a, 3e). We also examined the effect of lung-MSCs' secretome on the levels of peIF4E and peIF4GI. Here too, we observed
(healthy/NSCLC) secretome. After $72 \mathrm{~h}$, the cells were assayed for c cell number (Trypan blue), migration (scratch-24h), and d immunoblotted for TI factors, PCNA/cyclin D1 proliferation markers. Results are presented as percent $($ mean \pm SE) of control cells (dotted line) and normalized to HSC-70 loading control $(n \geq 3)$. Representative immunoblots are presented (Fig. 3c, d). Statistically significant differences between cohorts $(* p<0.05$, **p $p<0.01$; compare to control. ${ }^{\$}$ Normal vs. NSCLC) are indicated

increased phosphorylated active levels of the TI factors (peIF4E: $100-140 \% \uparrow$, peIF4GI: $140 \% \uparrow ; p<0.05,1.5 \mathrm{~h}$ ) (Figs. 5a, 3e). Our results indicate that lung-MSCs' secretome elevates MAPKs, eIF4E/eIF4GI, and migration.

Next, in order to establish the regulatory connection between MAPKs signals, eIF4E/eIF4GI phosphorylation, and NSCLCs' migration, we treated NSCLC cells with lungMSCs' secretome in combination with JNK and ERK inhibitors (SP600125 and U0126, respectively). Our analyses demonstrated that the co-treatment decreased levels of phosphorylated TI factors in comparison to secretome-only treated cells $(94-130 \% \downarrow, p<0.05)$ following $1.5 \mathrm{~h}$ (Figs. 5b, 3f). We validated the inhibitors' activity by assaying the 


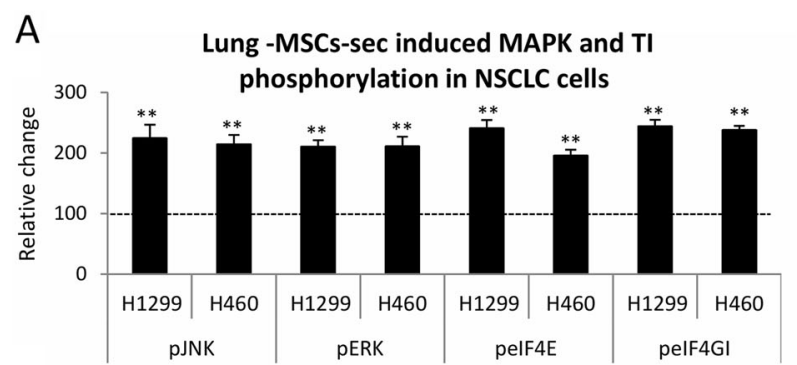

C MAPK signaling regulates migration of NSCLC cells
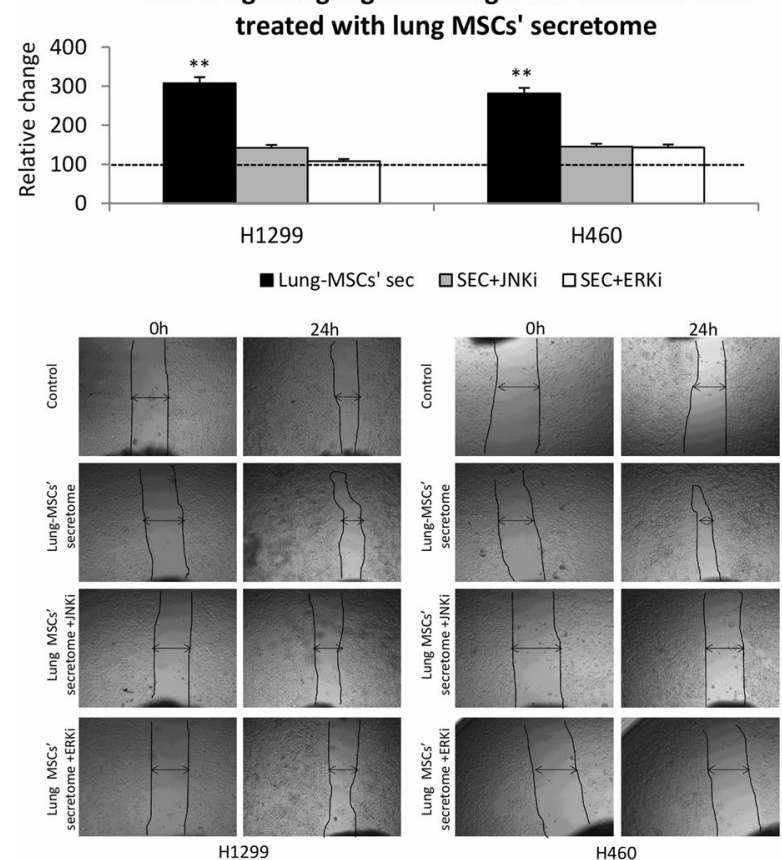

Fig. 5 Lung-MSCs secretome affects MAPKs/TI-dependent migration in NSCLC cell lines: a NSCLC cells were cultured with lung-MSCs secretome for $1.5 \mathrm{~h}$ and then were lysed and immonubloted for phosphorylated MAPK (JNK, ERK) and phosphorylated TI factors (eIF4E, eIF4GI). b, c MAPKs' inhibitors affected the TI factors' phosphorylation and cell migration in NSCLC cells treated with lungMSCs' sec: NSCLC cell lines were co-treated with lung-MSCs secretome and MAPK inhibitors (JNK inhibitor (SP600125, $20 \mu \mathrm{M}$, gray) and ERK inhibitor (U0126, $10 \mu \mathrm{M}$, white)). b After $1.5 \mathrm{~h}$, the cells were collected and immunoblotted for peIF4E/peIF4GI. c Next, the effect of MAPKs on NSCLC cells' migration was assessed by scratch assay. The cells were treated with the lung-MSCs' sec and MAPK inhibitors and scratch closure were photographed immediately

levels of the respective phosphorylated MAPKs (data not shown). Finally, we performed scratch assays in lungMSCs' secretome-treated NSCLC cell lines while JNK and ERK were inhibited. As expected, we determined reduced motility rates of lung-MSCs secretome-treated NSCLC cells upon either MAPKs' inhibition compared to secretome-only treatment $(140-200 \% \downarrow, p<0.05)$ (Fig. 5c). Of note, we have also witnessed reduced viability in NSCLC cells treated with combination of lung-MSCs' secretome and MAPK inhibitors showed in comparison to secretome-only treated cells $(75 \% \downarrow, p<0.05)$.
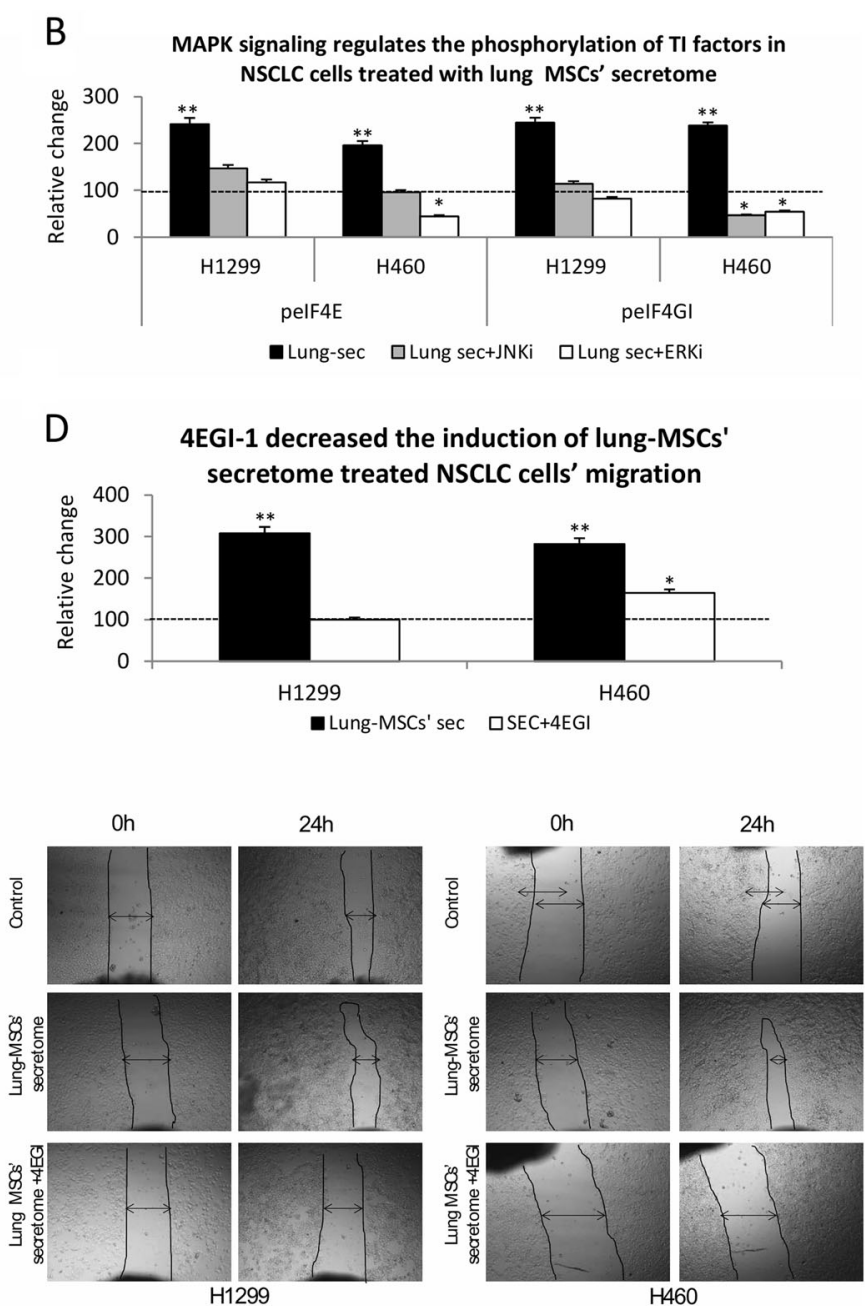

$(0 \mathrm{~h})$ and after $24 \mathrm{~h}$ (magnification, $\times 40)$. d Disassociation of eIF4E/ eIF4GI complex annulled the induction of lung-MSCs' secretome: lung-MSCs' secretome-treated NSCLC cells were treated with the small molecule 4EGI-1 (inhibit eIF4E/eIF4GI association $(35 \mu \mathrm{M})$ ). The effect of 4EGI-1 on NSCLC cells' migration was assessed by scratch assay. In all experiments, scratch closure were photographed immediately $(0 \mathrm{~h})$ and after $24 \mathrm{~h}$ (magnification, $\times 40)$. Results are presented as percent in bar graphs (top) and representative images. All results are presented in graphs as percent (mean $\pm \mathrm{SE})$ of control cells (dotted line) and immunoblot results were normalized to HSC-70 loading control $(n \geq 3)$. Representative immunoblots are presented (Fig. 3e, f). Statistically significant differences between cohorts $\left({ }^{*} p<\right.$ $0.05, * * p<0.01)$ are indicated

\section{Disassociation of elF4E/elF4GI complex annulled the induction of lung-MSCs' secretome-treated NSCLC cells' migration and viability}

We have previously substantiated that TI was critical for NSCLC cells' migration and demonstrated that eIF4E/ eIF4GI's activity is crucial for NSCLC migration. In the current study, we wanted to assay if that inhibition can annul the induction of NSCLC cells' migration and elevated viability we have witnessed following lung-MSCs' secretome treatment. For this purpose we used the small 


\section{A \\ Lung-MSCs' secretome induced EMT while BM-MSCs' secretome encouraged MET in NSCLC cells}

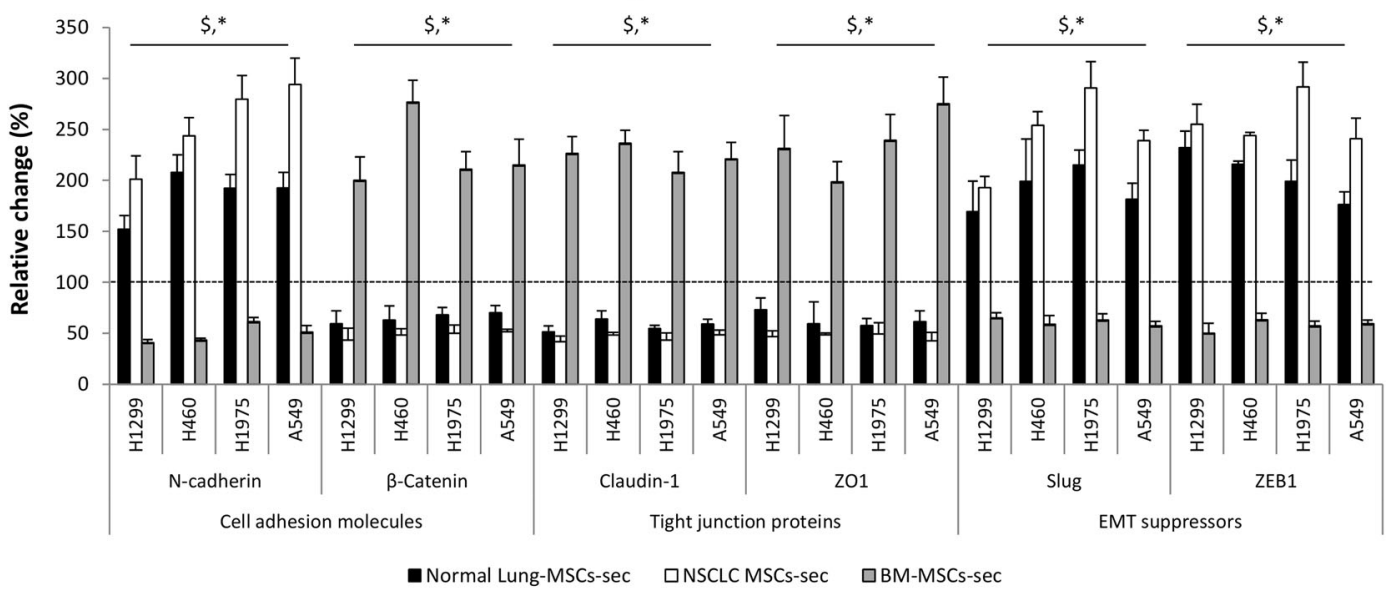

B

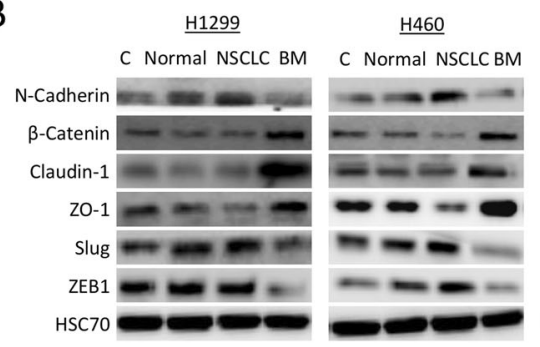

Fig. 6 MSCs' secretome induced EMT/MET signals in NSCLC cell lines: NSCLC cells were cultured with MSCs secretome (normal lung, NSCLC, bone marrow) for $72 \mathrm{~h}$ and then lysed and immonubloted for the EMT signals: N-cadherin, $\beta$-catenin, claudin-1, ZO1, Slug, and ZEB1. Results are presented as percent (mean \pm SE) of control cells (dotted line) and normalized to HSC-70 loading control ( $n \geq 3)$. Bar graphs (a) and representative Immunoblots (b) are presented. Statistically significant differences between cohorts $(* p<0.05, * * p<0.01$; compare to control. ${ }^{\$} \mathrm{BM}$ vs. normal vs. NSCLC) are indicated molecule 4EGI-1 inhibitor of TI that disrupts eIF4E/eIF4G association and performed scratch assay. We compared the scratch closure of NSCLC cells treated with lung-MSCs' secretome with and without 4EGI-1 and measured reduced motility rates of NSCLC cells upon eIF4E/eIF4GI disassociation $(120-200 \% \downarrow, p<0.05)$ (Fig. 5d). These findings suggest that the secretomes' manipulation of NSCLC migration is indeed contingent on TI pathways. Analysis of NSCLC cells viability upon treatment with lung-MSCs' secretomes with and without 4EGI-I also showed dependence on TI factors $(20 \% \downarrow, p<0.05)$.

\section{Lung-MSCs' secretome induced epithelial to mesenchymal transition while BM-MSCs' secretome encouraged mesenchymal to epithelial transition in NSCLC cells}

Based on our accumulated observations here and in our previous study [14], we hypothesized that the NSCLC exposed to secretomes of primary niche healthy lung-/NSCLC-MSCs or BM-MSCs undergo epithelial to mesenchymal transition
(EMT) or mesenchymal to epithelial transition (MET), respectively, via the modulation of TI. In order to assess the relevance of this conjecture to the effects of MSCs on NSCLC, we examine the expression of EMT/MET markers. First, we examined changes in cell-cell adhesion molecules $\mathrm{N}$-cadherin and $\beta$-catenin known to play a critical role in the EMT/MET. In concordance with our hypothesis, we determined elevated $\beta$-catenin (113-175\% $\uparrow)$ and reduced $\mathrm{N}$-cadherin $(40-60 \% \downarrow)$ in BM-MSCs' secretome treatment and the opposite effect in lung-MSCs' secretome treatment ( $\beta$-catenin: 30-60\% $\downarrow$; N-cadherin: 50-190 \%个) (Fig. 6a, b). Next, we assayed the tight junction-associated proteins claudin-1 and zona occludens-1 (ZO-1) implicated in EMT due to their role in cell-cell junctions and migration [29]. Again, we observed the same opposing effects of lung-MSCs and BM-MSCs secretomes (BM: 100-130\% $\uparrow$, lung: 30-60\% $\downarrow$; $p<0.05$ ) (Fig. 6a, b). Finally, we assessed the levels of two proteins that promote EMT: Slug, a transcriptional repressor of integrin that causes decreased cell adhesion [30] and ZEB1 transcription factor that suppress E-cadherin [31]. Examination of these two signals once again supported 
the EMT induction in lung-MSCs treatment and MET

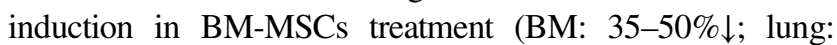
70-190\% $\uparrow p<0.05$ ) (Fig. 6a, b). Altogether, our data indicate that the MSCs of each niche are characterized with their own unique secretome that modulates EMT/MET response in NSCLC cells and consequently alters the malignant cells' phenotype.

\section{Discussion}

In this study, we have demonstrated that lung-MSCs stimulate NSCLC cells' proliferation and migration in a MAPK/TI-dependent manner (timeline is summarized in Fig. 7). We have also determined that the MSCs adjacent to the NSCLC tumor affect the NSCLC cell lines' phenotype more prominently than the distant lung-MSCs (healthy). While it is well established by others and us that the tumor microenvironment plays a pivotal role in cancer progression, the distinction between adjacent and distant lungMSCs is new [11, 32-35]. These findings are particularly significant when aligned with our publications regarding the inhibitory effect of BM-MSCs secretome on the same NSCLC cells. We propose that our observations can be categorized as effects of the primary tumor niche (lung) vs. the metastatic site (BM). In our studies, we have addressed the role of $\mathrm{BM}$ as the metastatic compartment, yet other metastatic sites are involved in the spread of lung cancer such as brain and adrenal glands. The study of these metastatic sites demonstrated mechanisms of NSCLC cell recruitment by CCL20/CCR6 in adrenal glands or Wnt signaling in brain microglia cells, which promoted invasion and colonialization [36, 37]. To the best of our knowledge, there has been no previous observation of the metastatic site MSCs' participation in the process.

In context of cancer progression, the primary niche encourages tumor expansion and dissemination, whereas the metastatic niche downregulates migration and allows the settlement of the cells in the bone (supplementary table 1). Generally, our findings in the current and previous work conform to this perception and delineate an active role for the respective niche MSCs in the design of the cancer cells characteristics, primary or metastatic. The mechanism underlying the epithelium-derived carcinoma cells' behavior at both niches is most often the developmental program termed EMT and its reverse program, MET [38]. While EMT occurs at the primary tumor site allowing the cancer epithelial cells' to gain mesenchymal characteristics and migrate to distant organs, MET is believed to participate in the establishment and stabilization of distant metastases by allowing cancerous cells to reclaim epithelial properties and settle [39]. Our results match this current understanding: primary tumor resident MSCs induced EMT of NSCLC

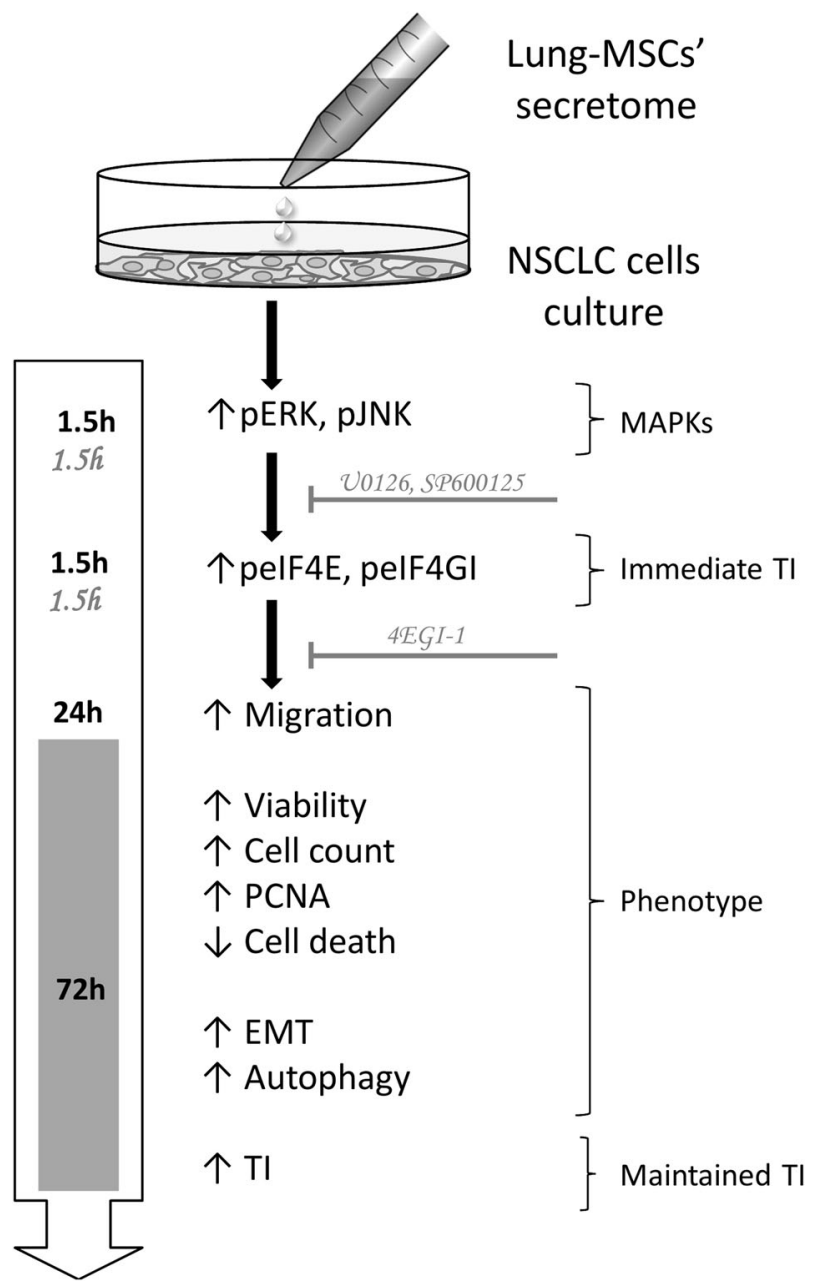

Fig. 7 Timeline of lung-MSCs' secretome effect on NSCLC cells: the figure presents the signaling activation sequence and phenotypic alterations following exposure of NSCLC cells to lung-MSCs' secretome. The schematic presentation describes the timeline of events that emerged from lung-MSCs' secretome, while the thin arrows indicate the trend of change. The gray lines represent the inhibitor effects at the same points. The left arrow depicts the time in which the effects were detected. Here too, the black font represent the time of the effects in the cells that were exposed to the lung-MSCs' secretome, while the gray font is the time for cells treated with the inhibitors. On the right side of the schema specified what was affected in each time point

cells while the same type of cells originating from the metastatic niche encourage MET phenotype. These findings unequivocally show that the MSCs role in cancer is nichedependent demonstrating their heterogeneity and conditioning by context.

Metastatic diseases are responsible for $>90 \%$ of carcinoma-related deaths. Given the strong evidence supporting a critical role of EMT/MET processes in tumor metastasis formation, targeting these processes is thought to be a promising approach to treat invasive cancers such as NSCLC. However, current treatment modalities remain limited in their efficacy in targeting cells undergoing EMT 
due to lack of appropriate EMT targets. Moreover, development of drug resistance over time and course of treatment is a major obstacle in cancer therapy and an increasing number of reports suggest a role for EMT in its acquisition since recurrent tumors frequently exhibit an EMT gene signature [40]. Shintani et al. [41] found that tumor biopsy in NSCLC patients prior to chemotherapy treatment showed epithelial markers, whereas there was a phenotypic shift toward mesenchymal markers following treatment. Interestingly, recent studies have also suggested a role for EMT in targeted therapies. Using several human NSCLC lines, Thomson et al. [42] demonstrated that cell lines expressing epithelial markers were more sensitive to epidermal growth factor receptor inhibition, while cell lines presenting mesenchymal markers were more resistant to treatment. In gefitinib-resistant PC9/AB2 lung cancer cells, Notch1 was found to promote EMT. Knockdown of Notch1 reverted the EMT phenotype back to epithelial and rendered these cells sensitive to gefitinib, implicating a strong correlation between EMT activation and drug resistance [43]. Taken together, these observations substantiate the need to devise means to maintain/revert the cancer cells to their epithelial phenotype in their primary niche.

Based on our findings, we put forward that interrupting the cross-talk between the cancer cells and the secreted factors from MSCs in both the primary and metastatic niches, may facilitate a control over the EMT/MET process and allow some control of metastasis formation in lung cancer patients' bone leading to improved survival and quality of life.

This study's observations have illuminated another important aspect regarding tumor microenvironment. We compared the effect of healthy MSCs' to NSCLC-MSCs' secretomes and discovered that although both secretomes supported the cancerous processes, the cells' response to the pathological secretome was more intense. These results are in concordance with previously published observations regarding the propensity of MSCs to home to tumors and their involvement in the malignant process [44]. Moreover, our findings accentuate the importance of NSCLC and MSCs cross-talk to recruitment and subversion of MSC into cancer-supporting participants with stage/location appropriate function. Another interesting alteration we have witnessed was elevated autophagy, which supported the NSCLC cells' survival. The role of autophagy and its protective function in NSCLC has been extensively discussed [26]. In concordance, our results implied that lung-MSCs' secretome protected against apoptosis by enhancing autophagy in NSCLC cells, which underscores autophagy targeting as anti-NSCLC/cancer treatment. Additional studies are needed to establish the role of autophagy manipulation in MSCs-NSCLC cross-talk and the clinical potential of its targeting.
Our preliminary studies with the secretome fractions support the existence of components within the $>100 \mathrm{kDa}$ fraction that affect the TI machinery in NSCLC cells while the $<100 \mathrm{kDa}$ fraction had no effect. Accumulating evidence indicates the involvement of MVs (present in the $>100 \mathrm{kDa}$ fraction) in cancer progression and specifically in NSCLC [45-47]. This very intriguing finding warrants further studies already underway by our group. A characterization of the lung-MSCs' MVs influence on NSCLC cells and examination of the MVs' cargoes may emphasize specific factors that shape the disease progression.

This study investigated the contribution of lung resident MSCs from normal lung and tumor adjacent tissue to NSCLC progression. Importantly, our observations were analyzed in comparison to those attributed to MSCs of the BM metastatic niche established by us previously [11] (supplementary table 1). We compiled explicit data indicating that lung-MSCs differ from that of the metastatic bone MSCs. This is critical to the understanding of the cancerous systemic state. Intrigued by our findings, ongoing studies in our lab are designed to locate specific soluble participants in the MSCs $>100 \mathrm{kDa}$ secretomes capable of modulating the cancer cells with the purpose of identifying targetable signals that will allow us to control the metastatic progression in NSCLC.

Acknowledgements This work was supported by the Cancer Biology Research Center (CBRC) \#0601242482 Dream Idea Grant, Tel Aviv University.

\section{Compliance with ethical standards}

Conflict of interest The authors declare that they have no conflict of interest.

\section{References}

1. Legrier ME, Yang CP, Yan HG, Lopez-Barcons L, Keller SM, Perez-Soler $\mathrm{R}$, et al. Targeting protein translation in human non small cell lung cancer via combined MEK and mammalian target of rapamycin suppression. Cancer Res. 2007;67:11300-8.

2. Chen Z, Fillmore CM, Hammerman PS, Kim CF, Wong KK. Non-small-cell lung cancers: a heterogeneous set of diseases. Nat Rev Cancer. 2014;14:535-46.

3. Balkwill FR, Capasso M, Hagemann T. The tumor microenvironment at a glance. J Cell Sci. 2012;125(Pt 23):5591-6.

4. Quail DF, Joyce JA. Microenvironmental regulation of tumor progression and metastasis. Nat Med. 2013;19:1423-37.

5. Korkaya H, Wicha MS. Breast cancer stem cells: we've got them surrounded. Clin Cancer Res. 2013;19:511-3.

6. Serakinci N, Tulay P, Kalkan R. Role of mesenchymal stem cells in cancer development and their use in cancer therapy. Adv Exp Med Biol. Springer, Boston, MA 2017.

7. Ullah I, Subbarao RB, Rho GJ. Human mesenchymal stem cells current trends and future prospective. Biosci Rep. 2015;35: e00191.

8. Yagi H, Kitagawa Y. The role of mesenchymal stem cells in cancer development. Front Genet. 2013;4:261. 
9. Steeg PS. Targeting metastasis. Nat Rev Cancer. 2016;16:201-18.

10. Yoneda T, Hiraga T. Crosstalk between cancer cells and bone microenvironment in bone metastasis. Biochem Biophys Res Commun. 2005;328:679-87.

11. Attar-Schneider O, Zismanov V, Drucker L, Gottfried M. Secretome of human bone marrow mesenchymal stem cells: an emerging player in lung cancer progression and mechanisms of translation initiation. Tumour Biol. 2016;37:4755-65.

12. Klaus M, Stavroulaki E, Kastrinaki MC, Fragioudaki P, Giannikou K, Psyllaki M, et al. Reserves, functional, immunoregulatory, and cytogenetic properties of bone marrow mesenchymal stem cells in patients with myelodysplastic syndromes. Stem Cells Dev. 2010;19:1043-54.

13. Gottschling S, Granzow M, Kuner R, Jauch A, Herpel E, Xu EC, et al. Mesenchymal stem cells in non-small cell lung cancer-different from others? Insights from comparative molecular and functional analyses. Lung Cancer. 2013;80:19-29.

14. Attar-Schneider O, Drucker L, Gottfried M. Migration and epithelial-to-mesenchymal transition of lung cancer can be targeted via translation initiation factors eIF4E and eIF4GI. Lab Investig. 2016;96:1004-15.

15. Zismanov V, Lishner M, Tartakover-Matalon S, Radnay J, Shapiro H, Drucker L. Tetraspanin-induced death of myeloma cell lines is autophagic and involves increased UPR signalling. $\mathrm{Br} \mathrm{J}$ Cancer. 2009;101:1402-9.

16. Attar-Schneider O, Drucker L, Zismanov V, Tartakover-Matalon S, Rashid G, Lishner M. Bevacizumab attenuates major signaling cascades and eIF4E translation initiation factor in multiple myeloma cells. Lab Invest. 2012;92:178-90.

17. Zismanov V, Drucker L, Gottfried M. ER homeostasis and motility of NSCLC cell lines can be therapeutically targeted with combined Hsp90 and HDAC inhibitors. Pulm Pharmacol Ther. 2013;26:388-94.

18. O'Hayre M, Salanga CL, Handel TM, Allen SJ. Chemokines and cancer: migration, intracellular signalling and intercellular communication in the microenvironment. Biochem $\mathrm{J}$. 2008;409:635-49.

19. Jumper C, Cobos E, Lox C. Determination of the serum matrix metalloproteinase-9 (MMP-9) and tissue inhibitor of matrix metalloproteinase-1 (TIMP-1) in patients with either advanced small-cell lung cancer or non-small-cell lung cancer prior to treatment. Respir Med. 2004;98:173-7.

20. Leinonen T, Pirinen R, Bohm J, Johansson R, Kosma VM. Increased expression of matrix metalloproteinase-2 (MMP-2) predicts tumour recurrence and unfavourable outcome in nonsmall cell lung cancer. Histol Histopathol. 2008;23:693-700.

21. Deryugina EI, Quigley JP. Matrix metalloproteinases and tumor metastasis. Cancer Metastasis Rev. 2006;25:9-34.

22. Dolfi SC, Chan LL, Qiu J, Tedeschi PM, Bertino JR, Hirshfield $\mathrm{KM}$, et al. The metabolic demands of cancer cells are coupled to their size and protein synthesis rates. Cancer Metab. 2013;1:20.

23. Zismanov V, Drucker L, Attar-Schneider O, Matalon ST, Pasmanik-Chor M, Lishner M. Tetraspanins stimulate protein synthesis in myeloma cell lines. $\mathrm{J}$ Cell Biochem. 2012;113:2500-10.

24. Thornton S, Anand N, Purcell D, Lee J. Not just for housekeeping: protein initiation and elongation factors in cell growth and tumorigenesis. J Mol Med. 2003;81:536-48.

25. Robert F, Pelletier J. Translation initiation: a critical signalling node in cancer. Expert Opin Ther Targets. 2009;13:1279-93.

26. Liu G, Pei F, Yang F, Li L, Amin AD, Liu S, et al. Role of autophagy and apoptosis in non-small-cell lung cancer. Int J Mol Sci. 2017;18.

27. Lau MT, So WK, Leung PC. Fibroblast growth factor 2 induces E-cadherin down-regulation via $\mathrm{PI} 3 \mathrm{~K} / \mathrm{Akt} / \mathrm{mTOR}$ and MAPK/
ERK signaling in ovarian cancer cells. PLoS ONE. 2013;8: e59083.

28. Shveygert M, Kaiser C, Bradrick SS, Gromeier M. Regulation of eukaryotic initiation factor 4E (eIF4E) phosphorylation by mitogen-activated protein kinase occurs through modulation of Mnk1-eIF4G interaction. Mol Cell Biol. 2010;30:5160-7.

29. Polakis P. The oncogenic activation of beta-catenin. Curr Opin Genet Dev. 1999;9:15-21.

30. Turner FE, Broad S, Khanim FL, Jeanes A, Talma S, Hughes S, et al. Slug regulates integrin expression and cell proliferation in human epidermal keratinocytes. J Biol Chem. 2006; 281:21321-31.

31. Aigner K, Dampier B, Descovich L, Mikula M, Sultan A, Schreiber M, et al. The transcription factor ZEB1 (deltaEF1) promotes tumour cell dedifferentiation by repressing master regulators of epithelial polarity. Oncogene. 2007;26:6979-88.

32. Marcus H, Attar-Schneider O, Dabbah M, Zismanov V, Tartakover-Matalon S, Lishner M, et al. Mesenchymal stem cells secretomes' affect multiple myeloma translation initiation. Cell Signal. 2016;28:620-30.

33. Dabbah M, Attar-Schneider O, Zismanov V, Tartakover Matalon S, Lishner M, Drucker L. Multiple myeloma cells promote migration of bone marrow mesenchymal stem cells by altering their translation initiation. J Leukoc Biol. 2016;100:761-70.

34. Dabbah M, Attar-Schneider O, Tartakover Matalon S, Shefler I, Jarchwsky Dolberg O, Lishner M, et al. Microvesicles derived from normal and multiple myeloma bone marrow mesenchymal stem cells differentially modulate myeloma cells' phenotype and translation initiation. Carcinogenesis. 2017;38:708-16.

35. Attar-Schneider O, Zismanov V, Dabbah M, Tartakover-Matalon S, Drucker L, Lishner M. Multiple myeloma and bone marrow mesenchymal stem cells' crosstalk: effect on translation initiation. Mol Carcinog. 2016;55:1343-54.

36. Pukrop T, Dehghani F, Chuang HN, Lohaus R, Bayanga K, Heermann S, et al. Microglia promote colonization of brain tissue by breast cancer cells in a Wnt-dependent way. Glia. 2010;58:1477-89.

37. Raynaud CM, Mercier O, Dartevelle P, Commo F, Olaussen KA, de Montpreville $\mathrm{V}$, et al. Expression of chemokine receptor CCR6 as a molecular determinant of adrenal metastatic relapse in patients with primary lung cancer. Clin Lung Cancer. 2010;11:187-91.

38. Tsai JH, Yang J. Epithelial-mesenchymal plasticity in carcinoma metastasis. Genes Dev. 2013;27:2192-206.

39. Campolo F, Gori M, Favaro R, Nicolis S, Pellegrini M, Botti F, et al. Essential role of Sox 2 for the establishment and maintenance of the germ cell line. Stem Cells. 2013;31:1408-21.

40. Kawamoto A, Yokoe T, Tanaka K, Saigusa S, Toiyama Y, Yasuda $\mathrm{H}$, et al. Radiation induces epithelial-mesenchymal transition in colorectal cancer cells. Oncol Rep. 2012;27:51-7.

41. Shintani Y, Okimura A, Sato K, Nakagiri T, Kadota Y, Inoue M, et al. Epithelial to mesenchymal transition is a determinant of sensitivity to chemoradiotherapy in non-small cell lung cancer. Ann Thorac Surg. 2011;92:1794-804.

42. Thomson S, Buck E, Petti F, Griffin G, Brown E, Ramnarine N, et al. Epithelial to mesenchymal transition is a determinant of sensitivity of non-small-cell lung carcinoma cell lines and xenografts to epidermal growth factor receptor inhibition. Cancer Res. 2005;65:9455-62.

43. Xie M, Zhang L, He CS, Xu F, Liu JL, Hu ZH, et al. Activation of Notch-1 enhances epithelial-mesenchymal transition in gefitinibacquired resistant lung cancer cells. $\mathrm{J}$ Cell Biochem. 2012;113:1501-13.

44. Reagan MR, Kaplan DL. Concise review: mesenchymal stem cell tumor-homing: detection methods in disease model systems. Stem Cells. 2011;29:920-7. 
45. D’Souza-Schorey C, Clancy JW. Tumor-derived microvesicles: shedding light on novel microenvironment modulators and prospective cancer biomarkers. Genes Dev. 2012;26:1287-99.

46. Zhang L, Valencia CA, Dong B, Chen M, Guan PJ, Pan L. Transfer of microRNAs by extracellular membrane microvesicles: a nascent crosstalk model in tumor pathogenesis, especially tumor cell-microenvironment interactions. J Hematol Oncol. 2015;8:14.

47. Wysoczynski M, Ratajczak MZ. Lung cancer secreted microvesicles: underappreciated modulators of microenvironment in expanding tumors. Int J Cancer. 2009;125:1595-603. 